International Journal of Pure and Applied Mathematics

Volume 101 No. 1 2015, 71-82

ISSN: 1311-8080 (printed version); ISSN: 1314-3395 (on-line version)

url: http://www.ijpam.eu

doi: http://dx.doi.org/10.12732/ijpam.v101i1.7

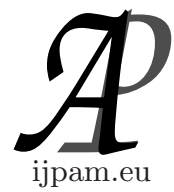

\title{
GENERALIZED FRACTIONAL INTEGRAL INEQUALITIES INVOLVING HYPERGEOMETRIC OPERATORS
}

\author{
Seda Kilinç ${ }^{1 \S}$, Hüseyin Yildirim² \\ ${ }^{1}$ Department of Mathematics \\ Faculty of Science and Arts \\ University of Kahramanmaras \\ Sütçü İmam, 46000, Kahramanmaraş, TURKEY
}

\begin{abstract}
In this paper we establish new generalized fractional integral inequalities involving the Gauss hypergeometric function for synchronous functions which are related to the Chebyshev functional.We deal with generalized fractional integral inequalities involving Saigo, Erdelyi-Kober and RiemannLiouville type fractional integral operators.
\end{abstract}

AMS Subject Classification: 26A33, 26D10, 26D15, 41A55

Key Words: fractional integral, Riemann-Liouville fractional integral, hypergeometric function

\section{Introduction}

In recent years, much attention has been given to fractional integral inequalities. There are many applications, extensions and generalizations we refer to such type of studies in $[3,16]$. They provide upper and lower bounds to the solutions of fractional partial differential equations. The aim of this article is to obtain new integral inequalities for synchronous functions that are related to the Chebyshev functional using generalized $\alpha$-Riemann-Liouville $k$ - fractional integrals.

Received: January 29, 2015

(C) 2015 Academic Publications, Ltd.

$\S$ Correspondence author url: www.acadpubl.eu 


\section{Preliminaries}

In this section we will give some definitions and related details.

Definition 1. Two functions $f$ and $g$ are said to be synchronous on $[a, b]$, if

$$
\{(f(x)-f(y))(g(x)-g(y))\} \geq 0,
$$

for any $x, y \in[a, b]$,

Definition 2. A real-valued function $f(t)(t>0)$ is said to be in the space $\mathbb{C}_{\mu}(\mu \in \mathbb{R})$ if there exists a real number $p>\mu$ such that $f(t)=t^{p} \phi(t)$, where $\phi(t) \in \mathbb{C}(0, \infty)$.

Definition 3. [22] A function $f(t), t>0$ is said to be in the $L_{p, k}[0, \infty)$ space if

$$
\begin{aligned}
& L_{p, k[0, \infty)}= \\
& \quad\left\{f:\|f\|_{L_{p, k[0, \infty)}}=\left(\int_{0}^{\infty}|f(t)|^{p} t^{k} d t\right)^{\frac{1}{p}}<\infty, 1 \leq p<\infty, k \geq 0\right\} .
\end{aligned}
$$

Definition 4. [21] Let $f \in L_{1}[0, \infty)$.The Riemann- Liouville fractional integral of order $\alpha \geq 0$ is defined by

$$
\begin{gathered}
I^{\alpha} f(t)=\frac{1}{\Gamma(\alpha)} \int_{0}^{t}(t-x)^{\alpha-1} f(x) d x, \\
I^{0} f(t)=f(t)
\end{gathered}
$$

where $\Gamma$ is the gamma function.

Definition 5. $\quad[11,21,22]$ Let $f \in L_{1, k}[0, \infty)$. The Generalized RiemannLiouville fractional integral $I^{\alpha, k} f(x)$ of order $\alpha \geq 0$ and $k \geq 0$ is defined by

$$
\begin{gathered}
I^{\alpha, k} f(x)=\frac{(k+1)^{1-\alpha}}{\Gamma(\alpha)} \int_{0}^{x}\left(x^{k+1}-t^{k+1}\right)^{\alpha-1} t^{k} f(t) d t, \\
I^{0, k} f(x)=f(x),
\end{gathered}
$$

where $\Gamma$ is the gamma function. 
Definition 6. Let $k \geq 0, \alpha>0, \mu>-1$, and $\beta, \eta \in \mathbb{R}$; then a generalized $k$ - fractional integral $I_{t, k}^{\alpha, \widehat{\beta, \eta}, \mu}$ (in terms of the Gauss hypergeometric function) of order $\alpha$ for a real -valued continuous function $f(t)$ is defined by [4] (see also [15] )

$$
\begin{array}{r}
I_{t, k}^{\alpha, \beta, \eta, \mu}[f(t)]=\frac{(k+1)^{\mu+\beta+1} t^{(k+1)(-\alpha-\beta-2 \mu)}}{\Gamma(\alpha)} \int_{0}^{t} \tau^{(k+1) \mu}\left(t^{k+1}-\tau^{k+1}\right)^{\alpha-1} \\
\times{ }_{2} F_{1}\left(\alpha+\beta+\mu,-\eta ; \alpha ; 1-\left(\frac{\tau}{t}\right)^{k+1}\right) \tau^{k} f(\tau) d \tau .
\end{array}
$$

where the functions ${ }_{2} F_{1}(-)$ appearing as a kernel for the operator (2.5) is the Gaussian hypergeometric function defined by

$$
{ }_{2} F_{1}(a, b ; c ; t)=\sum_{n=0}^{\infty} \frac{(a)_{n}(b)_{n}}{(c)_{n}} \frac{t^{n}}{n !} .
$$

The Pochhammer symbol $(a)_{n}$ is defined by:

$$
(a)_{n}=a(a+1) \ldots(a+n+1), \quad(a)_{0}=1 .
$$

For $\mu=0, \beta=-\alpha$ we obtain equality (2.4) in Definition 5 .

Also, for $k=0, \mu=0$, and $\beta=-\alpha$ we obtain equality (2.3) in Definition 4.

The aim of the present research is to obtain certain Chebyshev type integral inequalities including the generalized fractional integral operators [5] which involves the kernel, Gauss hypergeometric function (defined above). The concluding part gives some special state of the main results.

Let $k=0$ in (2.4) and (2.5), then we have the results in [3].

\section{Main Results}

In this section we obtain certain Chebyshev type integral inequalities involving the generalized $k$-fractional integral operator. The following lemma is used for our first result.

Lemma 1. Let, $k \geq 0, \alpha, \beta, \eta, \lambda \in \mathbb{R}, \mu>-1, \mu+\lambda>0$, and $\lambda-\beta+\eta>0$, then the following equality holds for symbol formula for (2.5) fractional integral.

$$
\begin{aligned}
& I_{t, k}^{\alpha, \beta, \eta, \mu}\left[t^{\lambda-1}\right] \\
& \quad=(k+1)^{\mu+\beta} t^{(k+1)(\lambda-\beta-\mu-1)} \frac{\Gamma\left(\mu+\frac{\lambda-1}{k+1}+1\right) \Gamma\left(\frac{\lambda-1}{k+1}+\eta-\beta+1\right)}{\Gamma\left(\frac{\lambda-1}{k+1}+1-\beta\right) \Gamma\left(\alpha+\mu+1+\frac{\lambda-1}{k+1}+\eta\right)} .
\end{aligned}
$$


Proof. If we take $f(\tau)=\tau^{\lambda-1}$ in (2.5) fractional integral, then we obtain,

$$
\begin{aligned}
& I_{t, k}^{\alpha, \beta, \eta, \mu}[f(t)]=\frac{(k+1)^{\mu+\beta+1} t^{(k+1)(-\alpha-\beta-2 \mu)}}{\Gamma(\alpha)} \\
& \quad \times \int_{0}^{t} \tau^{(k+1) \mu}\left(t^{k+1}-\tau^{k+1}\right)^{\alpha-1}{ }_{2} F_{1}\left(\alpha+\beta+\mu,-\eta ; \alpha ; 1-\left(\frac{\tau}{t}\right)^{k+1}\right) \tau^{k+\lambda-1} d \tau .
\end{aligned}
$$

If we use definition of Gaussian hypergeometric function, then we get, [14]

$$
\begin{aligned}
& \frac{(k+1)^{\mu+\beta+1} t^{(k+1)(-\alpha-\beta-2 \mu)}}{\Gamma(\alpha)} \\
& \times \int_{0}^{t} \tau^{(k+1) \mu}\left(t^{k+1}-\tau^{k+1}\right)^{\alpha-1}{ }_{2} F_{1}\left(\alpha+\beta+\mu,-\eta ; \alpha ; 1-\left(\frac{\tau}{t}\right)^{k+1}\right) \tau^{k+\lambda-1} d \tau \\
& =\frac{(k+1)^{\mu+\beta+1} t^{(k+1)(\mu+\alpha+\lambda-1)}}{\Gamma(\alpha)} \frac{\Gamma\left(\mu+\frac{\lambda-1}{k+1}+1\right) \Gamma(\alpha) \Gamma\left(\frac{\lambda-1}{k+1}+\eta-\beta+1\right)}{(k+1) \Gamma\left(\frac{\lambda-1}{k+1}+1-\beta\right) \Gamma\left(\alpha+\mu+1+\frac{\lambda-1}{k+1}+\eta\right)} \\
& =(k+1)^{\mu+\beta} t^{(k+1)(\lambda-\beta-\mu-1)} \frac{\Gamma\left(\mu+\frac{\lambda-1}{k+1}+1\right) \Gamma\left(\frac{\lambda-1}{k+1}+\eta-\beta+1\right)}{\Gamma\left(\frac{\lambda-1}{k+1}+1-\beta\right) \Gamma\left(\alpha+\mu+1+\frac{\lambda-1}{k+1}+\eta\right)} .
\end{aligned}
$$

Then (3.2) reduces to $(3.1)$.

Now, we obtain integral inequalities for the synchronous functions involving the generalized fractional integral operator (2.5).

Theorem 1. Let $f$ and $g$ be two synchronous functions on $[0, \infty)$. Then the following inequality holds for all $k \geq 0, t>0, \alpha>\max \{0,-\beta-\mu\}, \beta<1$, $\mu>-1, \beta-1<\eta<0$,

$$
\begin{aligned}
& I_{t, k}^{\alpha, \beta, \eta, \mu}[f(t) g(t)] \\
\geq & (k+1)^{-\mu-\beta} t^{(k+1)(\mu+\beta)} \frac{\Gamma(1-\beta) \Gamma(\alpha+\mu+\eta+1)}{\Gamma(1+\mu) \Gamma(1-\beta+\eta)} I_{t, k}^{\alpha, \beta, \eta, \mu}[f(t)] I_{t, k}^{\alpha, \beta, \eta, \mu}[g(t)] .
\end{aligned}
$$

Proof. Since $f$ and $g$ be two synchronous functions; from Definition 1 , for all $\tau, p \in(0, t), t \geq 0$, we have

$$
\{(f(\tau)-f(p))(g(\tau)-g(p))\} \geq 0
$$

which implies that

$$
f(\tau) g(\tau)+f(p) g(p) \geq f(\tau) g(p)+f(p) g(\tau) .
$$


Consider

$$
\begin{aligned}
& F(t, \tau)=\frac{(k+1)^{\mu+\beta+1} t^{(k+1)(-\alpha-\beta-2 \mu)}}{\Gamma(\alpha)} \tau^{(k+1) \mu}\left(t^{k+1}-\tau^{k+1}\right)^{\alpha-1}
\end{aligned}
$$

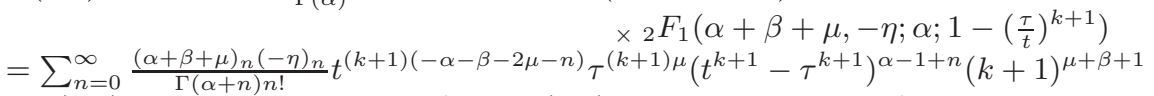

$$
\begin{aligned}
& =\frac{\tau^{(k+1) \mu}\left(t^{k+1}-\tau^{k+1}\right)^{\alpha-1}(k+1)^{\mu+\beta+1}}{t^{(k+1)(\alpha+\beta+2 \mu)} \Gamma(\alpha)}+\frac{\tau^{(k+1) \mu}\left(t^{k+1}-\tau^{k+1}\right)^{\alpha}(k+1)^{\mu+\beta+1}(\alpha+\beta+\mu)(-\eta)}{t^{(k+1)(\alpha+\beta+2 \mu+1)} \Gamma(\alpha+1)} \\
& +\frac{\tau^{(k+1) \mu}\left(t^{k+1}-\tau^{k+1}\right)^{\alpha+1}(k+1)^{\mu+\beta+1}(\alpha+\beta+\mu)(\alpha+\beta+\mu+1)(-\eta)(-\eta+1)}{t^{(k+1)(\alpha+\beta+2 \mu+2)} \Gamma(\alpha+2) 2 !}+\cdots
\end{aligned}
$$

Our observation is that each term of the above series is positive in view of the conditions stated with Theorem 1 , so the function $F(t, \tau)$ remains positive, for all $\tau \in(0, t) \quad(t>0)$.

Multiplying both sides of (3.6) by $F(t, \tau)$ (where $F(t, \tau)$ is given by (3.7) ) and integrating with respect to $\tau$ from 0 to $t$, and using (2.5), we have

$$
\begin{aligned}
I_{t, k}^{\alpha, \beta, \eta, \mu}[f(t) g(t)]+f(p) g(p) I_{t, k}^{\alpha, \beta, \eta, \mu} & {[1] } \\
& \geq g(p) I_{t, k}^{\alpha, \beta, \eta, \mu}[f(t)]+f(p) I_{t, k}^{\alpha, \beta, \eta, \mu}[g(t)] .
\end{aligned}
$$

Then, multiplying both sides of (3.8) by $F(t, p)(p \in(0, t), t>0)$, where $F(t, p)$ is given by (3.7) and integrating with respect to $p$ from 0 to $t$, and using Lemma 1 (for $\lambda=1$ ), we obtain designed result (3.4).

Theorem 2. Let $f$ and $g$ be two synchronous functions on $[0, \infty)$ Then the following inequality holds for all $k \geq 0, t>0, \alpha>\max \{0,-\beta-\mu\}, \gamma>$ $\max \{0,-\delta-\nu\}, \mu, \nu>-1, \beta, \delta<1, \beta-1<\eta<0, \delta-1<\zeta<0$,

$$
\begin{aligned}
& (k+1)^{\nu+\delta} t^{(k+1)(-\delta-\nu)} \frac{\Gamma(\nu+1) \Gamma(1-\delta+\zeta)}{\Gamma(1-\delta) \Gamma(\delta+\nu+\zeta+1)} I_{t, k}^{\alpha, \beta, \eta, \mu}[f(t) g(t)] \\
& +(k+1)^{\mu+\beta} t^{(k+1)(-\beta-\mu)} \frac{\Gamma(1+\mu) \Gamma(-\beta+\eta+1)}{\Gamma(1-\beta) \Gamma(\alpha+\mu+\eta+1)} I_{t, k}^{\gamma, \delta, \zeta, \nu}[f(t) g(t)] \\
& \geq I_{t, k}^{\gamma, \delta, \zeta, \nu}[f(t)] I_{t, k}^{\alpha, \beta, \eta, \mu}[g(t)]+I_{t, k}^{\gamma, \delta, \zeta, \nu}[g(t)] I_{t, k}^{\alpha, \beta, \eta, \mu}[f(t)]
\end{aligned}
$$

Proof. We use (3.8) inequality to prove the theorem. Multiplying both sides of (3.8) with,

$$
\begin{aligned}
& \frac{(k+1)^{\nu+\delta+1} t^{(k+1)(-\delta-\gamma-2 \nu)}}{\Gamma(\gamma)} p^{(k+1) \nu}\left(t^{k+1}-p^{k+1}\right)^{\gamma-1} \\
& \quad \times{ }_{2} F_{1}\left(\gamma+\delta+\nu,-\zeta ; \gamma ; 1-\left(\frac{p}{t}\right)^{k+1}\right) P(p), \quad(p \in(0, t) ; t>0)
\end{aligned}
$$

which remains positive in view of the conditions stated with (3.9) and integrating with respect to $p$ from 0 to $t$, we obtain 


$$
\begin{aligned}
I_{t, k}^{\gamma, \delta, \zeta, \nu}[1] I_{t, k}^{\alpha, \beta, \eta, \mu}[f(t) g(t)]+I_{t, k}^{\gamma, \delta, \zeta, \nu}[f(t) g(t)] I_{t, k}^{\alpha, \beta, \eta, \mu}[1] \\
\quad \geq I_{t, k}^{\gamma, \delta, \zeta, \nu}[f(t)] I_{t, k}^{\alpha, \beta, \eta, \mu}[g(t)]+I_{t, k}^{\gamma, \delta, \zeta, \nu}[g(t)] I_{t, k}^{\alpha, \beta, \eta, \mu}[f(t)]
\end{aligned}
$$

This result reduces to desired inequality by using (3.1) (for $\lambda=1$ ).

Remark 1. If may be said that inequalities (3.4) and (3.9) are reversed if the functions are asynchronous on $[0, \infty)$; that is,

$$
(f(x)-f(y))(g(x)-g(y)) \leq 0
$$

for any $x, y \in[0, \infty)$.

Remark 2. If we consider $\gamma=\alpha, \delta=\beta, \zeta=\eta$, and $\nu=\mu$, Theorem 2 we obtain that change to Theorem 1.

Theorem 3. Let $\left(f_{i}\right)_{i=1,2, \ldots, n}$ be $n$ positive increasing function on $[0, \infty)$. Then the following inequality holds for all $k \geq 0, t>0, \alpha>\max \{0,-\beta-\mu\}, \mu>$ $-1, \beta<1, \beta-1<\eta<0$

$$
\begin{aligned}
& I_{t, k}^{\alpha, \beta, \eta, \mu}\left[\prod_{i=1}^{n} f_{i}(t)\right] \\
& \geq\left[\frac{\Gamma(1-\beta) \Gamma(\alpha+\mu+\eta+1)}{\Gamma(1+\mu) \Gamma(1-\beta+\eta)} t^{(k+1)(\beta+\mu)}(k+1)^{-\mu-\beta}\right]^{n-1} \prod_{i=1}^{n} I_{t, k}^{\alpha, \beta, \eta, \mu}\left[f_{i}(t)\right] .
\end{aligned}
$$

Proof. To prove this theorem by mathematical induction.Clearly, for $n=1$ in $(3.13)$ we get

$$
I_{t, k}^{\alpha, \beta, \eta, \mu}\left[f_{1}(t)\right] \geq I_{t, k}^{\alpha, \beta, \eta, \mu}\left[f_{1}(t)\right]
$$

$(k \geq 0, t>0, \alpha>0)$, then for $n=2$ in (3.13), we have

$$
\begin{aligned}
I_{t, k}^{\alpha, \beta, \eta, \mu}\left[f_{1}(t) f_{2}(t)\right] \geq \frac{\Gamma(1-\beta) \Gamma(\alpha+\mu}{\Gamma(1+\mu) \Gamma(1-\beta+1)} & \beta+\eta) \\
& \times I_{t, k}^{\alpha, \beta, \eta, \mu}\left[f_{1}(t)\right] I_{t, k}^{\alpha, \beta, \eta, \mu}\left[f_{2}(t)\right],
\end{aligned}
$$

$(k \geq 0, t>0, \alpha>0)$ which holds in case of (3.4) of Theorem 1 .

By the induction principle, we consider that the inequality 


$$
\begin{aligned}
& I_{t, k}^{\alpha, \beta, \eta, \mu}\left[\prod_{i=1}^{n-1} f_{i}(t)\right] \\
& \geq\left[\frac{\Gamma(1-\beta) \Gamma(\alpha+\mu+\eta+1)}{\Gamma(1+\mu) \Gamma(1-\beta+\eta)} t^{(k+1)(\beta+\mu)}(k+1)^{-\mu-\beta}\right]^{n-2} \prod_{i=1}^{n-1} I_{t, k}^{\alpha, \beta, \eta, \mu}\left[f_{i}(t)\right],
\end{aligned}
$$

holds true for some positive integer $n \geq 2$.

Now $\left(f_{i}\right)_{i=1,2, \ldots, n}$ are increasing functions which show that the function $\prod_{n=1}^{n-1} f_{i}(t)$ is an increasing function too. Therefore, we can apply the inequality (3.4) of Theorem 1 to the functions $\prod_{n=1}^{n-1} f_{i}(t)=g$ and $f_{n}=f$ provided that $k \geq 0, t>0, \alpha>\max \{0,-\beta-\mu\}, \mu>-1, \beta<1, \beta-1<\eta<0$,

$$
\begin{array}{r}
I_{t, k}^{\alpha, \beta, \eta, \mu}\left[\prod_{i=1}^{n} f_{i}(t)\right] \geq \frac{\Gamma(1-\beta) \Gamma(\alpha+\mu+\eta+1)}{\Gamma(1+\mu) \Gamma(1-\beta+\eta)} t^{(k+1)(\beta+\mu)}(k+1)^{-\mu-\beta} \\
\times I_{t, k}^{\alpha, \beta, \eta, \mu}\left[\prod_{i=1}^{n-1} f_{i}(t)\right] I_{t, k}^{\alpha, \beta, \eta, \mu}\left[f_{n}(t)\right]
\end{array}
$$

Then if we use (3.16), we obtain desired inequality.

Now, we suppose another variation of the fractional integral inequalities.

Theorem 4. Let $f$ and $g$ be two functions on $[0, \infty)$ such that $f$ increasing $g$ is differentiable, and there exists a $P(t)$ polynomial $\inf _{t \geq 0}\left(\frac{g^{\prime}(t)}{P^{\prime}(t)}\right) \geq 1$. Then the following inequality holds for all $k \geq 0, t>0, \alpha>0, \mu>-1, \beta<1, \beta-1<$ $\eta<0$,

$$
\begin{aligned}
& I_{t, k}^{\alpha, \beta, \eta, \mu}[f(t) g(t)] \geq \frac{\Gamma(1-\beta) \Gamma(\alpha+\mu+\eta+1)}{\Gamma(1+\mu) \Gamma(1-\beta+\eta)} t^{(k+1)(\beta+\mu)}(k+1)^{-\mu-\beta} \\
& \quad \times I_{t, k}^{\alpha, \beta, \eta, \mu}[f(t)] I_{t, k}^{\alpha, \beta, \eta, \mu}[g(t)] \\
&-\frac{\Gamma(1-\beta) \Gamma(\alpha+\mu+\eta+1)}{\Gamma(1+\mu) \Gamma(1-\beta+\eta)} t^{(k+1)(\beta+\mu)}(k+1)^{-\mu-\beta} \\
& \quad \times I_{t, k}^{\alpha, \beta, \eta, \mu}[f(t)] I_{t, k}^{\alpha, \beta, \eta, \mu}[P(t)]+I_{t, k}^{\alpha, \beta, \eta, \mu}[f(t) P(t)] .
\end{aligned}
$$

Proof. When we consider the function $h(t)=g(t)-P(t)$. It is clear that $\mathrm{h}$ is differentiable and it is increasing on $[0, \infty)$; therefore, by using Theorem 1 , 
we have

$$
\begin{array}{r}
I_{t, k}^{\alpha, \beta, \eta, \mu}[f(t)(g(t)-P(t))] \geq \frac{\Gamma(1-\beta) \Gamma(\alpha+\mu+\eta+1)}{\Gamma(1+\mu) \Gamma(1-\beta+\eta)} t^{(k+1)(\beta+\mu)}(k+1)^{-\mu-\beta} \\
\times I_{t, k}^{\alpha, \beta, \eta, \mu}[f(t)] I_{t, k}^{\alpha, \beta, \eta, \mu}[g(t)-P(t)] \\
\geq \frac{\Gamma(1-\beta) \Gamma(\alpha+\mu+\eta+1)}{\Gamma(1+\mu) \Gamma(1-\beta+\eta)} t^{(k+1)(\beta+\mu)}(k+1)^{-\mu-\beta} \times I_{t, k}^{\alpha, \beta, \eta, \mu}[f(t)] I_{t, k}^{\alpha, \beta, \eta, \mu}[g(t)] \\
-\frac{\Gamma(1-\beta) \Gamma(\alpha+\mu+\eta+1)}{\Gamma(1+\mu) \Gamma(1-\beta+\eta)} t^{(k+1)(\beta+\mu)}(k+1)^{-\mu-\beta} \times I_{t, k}^{\alpha, \beta, \eta, \mu}[f(t)] I_{t, k}^{\alpha, \beta, \eta, \mu}[P(t)] \\
+I_{t, k}^{\alpha, \beta, \eta, \mu}[f(t) P(t)] .
\end{array}
$$

If we use, (3.1) (for $\lambda=2$ ) we have (3.18).

Theorem 5. Let $f$ and $g$ be two functions on $[0, \infty)$ such that $f$ increasing, $g$ is differentiable, and there exists a $p(t)$ polynomial $\sup _{t \geq 0}\left(\frac{g^{\prime}(t)}{p^{\prime}(t)}\right) \geq 1$. Then the following inequality holds for all $k \geq 0, t>0, \alpha>0, \mu>-1, \beta<1, \beta-1<$ $\eta<0$,

$$
\begin{aligned}
& I_{t, k}^{\alpha, \beta, \eta, \mu}[f(t) g(t)] \geq \frac{\Gamma(1-\beta) \Gamma(\alpha+\mu+\eta+1)}{\Gamma(1+\mu) \Gamma(1-\beta+\eta)} t^{(k+1)(\beta+\mu)}(k+1)^{-\mu-\beta} \\
& \times I_{t, k}^{\alpha, \beta, \eta, \mu}[f(t)] I_{t, k}^{\alpha, \beta, \eta, \mu}[g(t)] \\
&- \\
& \quad \frac{\Gamma(1-\beta) \Gamma(\alpha+\mu+\eta+1)}{\Gamma(1+\mu) \Gamma(1-\beta+\eta)} t^{(k+1)(\beta+\mu)}(k+1)^{-\mu-\beta} \\
& \quad \times I_{t, k}^{\alpha, \beta, \eta, \mu}[f(t)] I_{t, k}^{\alpha, \beta, \eta, \mu}[p(t)]+I_{t, k}^{\alpha, \beta, \eta, \mu}[f(t) p(t)] .
\end{aligned}
$$

Proof. By applying the similar arguments as in the proof of Theorem 4, it is easily to verify statement. Hence, we omitted the details of the proof.

Corollary 1. Let $f$ and $g$ be two functions on $[0, \infty)$ such that $f$ increasing $g$ is differentiable, and there exists a $P(t)$ polynomial $\inf _{t \geq 0}\left(\frac{g^{\prime}(t)}{P^{\prime}(t)}\right) \geq 1$. Then

$$
\begin{aligned}
& I_{0, t, k}^{\alpha, \beta, \eta}\{f(t) g(t)\}=I_{t, k}^{\alpha, \beta, \eta, 0}\{f(t) g(t)\} \\
& \geq \frac{\Gamma(1-\beta) \Gamma(\alpha+\eta+1)}{\Gamma(1-\beta+\eta)} t^{(k+1) \beta}(k+1)^{-\beta} I_{0, t, k}^{\alpha, \beta, \eta}\{f(t)\} I_{0, t, k}^{\alpha, \beta, \eta}\{g(t)\} \\
& -\frac{\Gamma(1-\beta) \Gamma(\alpha+\eta+1)}{\Gamma(1-\beta+\eta)} t^{(k+1) \beta}(k+1)^{-\beta} I_{0, t, k}^{\alpha, \beta, \eta}\{f(t)\} I_{0, t, k}^{\alpha, \beta, \eta}\{P(t)\}
\end{aligned}
$$




$$
+I_{0, t, k}^{\alpha, \beta, \eta}\{f(t) P(t)\}
$$

for all $k \geq 0, t>0, \alpha>0, \mu>-1, \beta<1, \beta-1<\eta<0$.

Corollary 2. Let $f$ and $g$ be two functions on $[0, \infty)$ such that $f$ increasing, $g$ is differentiable, and there exists a $p(t)$ polynomial $\sup _{t \geq 0}\left(\frac{g^{\prime}(t)}{p^{\prime}(t)}\right) \geq 1$

$$
\begin{aligned}
& I_{0, t, k}^{\alpha, \beta, \eta}\{f(t) g(t)\}=I_{t, k}^{\alpha, \beta, \eta, 0}\{f(t) g(t)\} \\
& \geq \frac{\Gamma(1-\beta) \Gamma(\alpha+\eta+1)}{\Gamma(1-\beta+\eta)} t^{(k+1) \beta}(k+1)^{-\beta} I_{0, t, k}^{\alpha, \beta, \eta}\{f(t)\} I_{0, t, k}^{\alpha, \beta, \eta}\{g(t)\} \\
& -\frac{\Gamma(1-\beta) \Gamma(\alpha+\eta+1)}{\Gamma(1-\beta+\eta)} t^{(k+1) \beta}(k+1)^{-\beta} I_{0, t, k}^{\alpha, \beta, \eta}\{f(t)\} I_{0, t, k}^{\alpha, \beta, \eta}\{p(t)\} \\
& +I_{0, t, k}^{\alpha, \beta, \eta}\{f(t) p(t)\}
\end{aligned}
$$

for all $k \geq 0, t>0, \alpha>0, \mu>-1, \beta<1, \beta-1<\eta<0$.

Corollary 3. Let $f$ and $g$ be two functions on $[0, \infty)$ such that $f$ increasing $g$ is differentiable, and there exists a $P(t)$ polynomial $\inf _{t \geq 0}\left(\frac{g^{\prime}(t)}{P^{\prime}(t)}\right) \geq 1$. Then the following inequality holds for all $k \geq 0, t>0, \alpha>0, \mu>-1, \beta<1, \beta-1<$ $\eta<0$,

$$
\begin{aligned}
& I_{t, k}^{\alpha, \eta}[f(t) g(t)]=I_{t, k}^{\alpha, 0, \eta, 0}[f(t) g(t)] \\
& \geq \frac{\Gamma(\alpha+\eta+1)}{\Gamma(1+\eta)} I_{0, t, k}^{\alpha, \eta}[f(t)] I_{0, t, k}^{\alpha, \eta}[g(t)] \\
& -\frac{\Gamma(\alpha+\eta+1)}{\Gamma(1+\eta)} I_{0, t, k}^{\alpha, \eta}[f(t)] I_{0, t, k}^{\alpha, \eta}[P(t)] \\
& +I_{0, t, k}^{\alpha, \eta}[f(t) P(t)] .
\end{aligned}
$$

Corollary 4. Let $f$ and $g$ be two functions on $[0, \infty)$ such that $f$ increasing, $g$ is differentiable, and there exists a $p(t)$ polynomial $\sup _{t \geq 0}\left(\frac{g^{\prime}(t)}{p^{\prime}(t)}\right) \geq 1$; Then the following inequality holds for all $k \geq 0, t>0, \alpha>0, \mu>-1, \beta<$ $1, \beta-1<\eta<0$

$$
\begin{aligned}
& I_{t, k}^{\alpha, \eta}[f(t) g(t)]=I_{t, k}^{\alpha, 0, \eta, 0}[f(t) g(t)] \\
& \geq \frac{\Gamma(\alpha+\eta+1)}{\Gamma(1+\eta)} I_{0, t, k}^{\alpha, \eta}[f(t)] I_{0, t, k}^{\alpha, \eta}[g(t)] \\
& -\frac{\Gamma(\alpha+\eta+1)}{\Gamma(1+\eta)} I_{0, t, k}^{\alpha, \eta}[f(t)] I_{0, t, k}^{\alpha, \eta}[p(t)]
\end{aligned}
$$




$$
+I_{0, t, k}^{\alpha, \eta}[f(t) p(t)] .
$$

As a result, if we take $\mu=0$ and $\beta=-\alpha(\nu=0$ and $\delta=-\gamma$ additionally for Theorem 2), then Theorems 1 to 5, yields the known results due to Belarbi and Dahmani [4].

\section{References}

[1] A. Akkurt, H. Yıldırım, "On Feng Qi Type Integral Inequalities For Generalized Fractional İntegrals" IAAOJ, Scientific Science, 1(2), (2013),22-25.

[2] G. A. Anastassiou, Advances on Fractional Inequalities, Springer Briefs in Mathematics, Springer, New York, NY, USA, (2011).

[3] D. Baleanu, S. D. Purohit, and Praveen Agarwal, "On Fractional Inequalities Involving Hypergeometric Operators" Chinese Journal of Mathematics,vol.2014 Article ID 609476, (2014), 5 pages.

[4] S.Belarbi and Z. Dahmani, "On some new fractional integral inequalities," Journal of Inequalities in Pure and Applied Mathematics, vol. 10, no. 3, article 86, 2009, 5 pages.

[5] L.Curiel and L.Galue, "A generalization of the integral operators involving the Gauss hypergeometric function," Revista Tècnica de la Facultad de Ingenierīa Universidad del Zulia,vol.19,no. 1, (1996), pp.17-22.

[6] P.L.Chebyshev, "Sur les expressions approximatives des integrales definies par les autres prises entre les měmes limities," Proceedings of the Mathematical Society of Kharkov, vol. 2 (1882), pp. 93-98.

[7] Z. Dahmani, O. Mechouar, and S. Brahami, "Certain inequalities related to the Chebyshev's functional involving a type Riemann-Lioville operator, " Bulletin of Mathematical Analysis and Applications, vol. 3, no. 4, (2011), pp. 38-44.

[8] Z. Denton and A. S. Vatsala, "Monotonic iterative technique for finit system of nonlinear Riemann-Liouville fractional differential equations, "Opuscula Mathematica, vol. 31, no. 3, (2011) pp. 327-339.

[9] S. S. Dragomir, "Some integral inequalities of Grüss type,"Indian Journal of Pure and Applied Mathematics, vol. 31, no. 4, (2000), pp. 397-415. 
[10] S. L. Kalla and A. Rao, "On Grüss type inequality for hypergeometric fractional integrals," Le Matematiche, vol. 66, no. 1,(2011), pp. 57-64.

[11] Katugampola, U.-N. New Approach to a generalized fractional integral, Appl. Math. Comput. 218(3), (2011), 860-865.

[12] V.S.Kiryakova, Generalized Fractional Calculus and Applications, Pitman Research Notes in Mathematics Series no. 301, Longman Scientific \& Technical, Harlow, UK, (1994).

[13] V. Lakshmikantham and A. S. Vatsala, "Theory of fractional differential inequalities and applications, "Communications in Applied Analysis, vol. 11, no.3-4, (2007), pp. 395-402.

[14] A. M. Mathai, R. K. Saxena, and H. J. Haubold, The H-Function: Theory and Applications, Springer, Dordrecht, The Netherlands, (2010).

[15] H. Öğ̈̈nmez and U. M. Özkan, "Fractional quantum integral inequalities," Journal of Inequalities and Applications, vol. 2011, Article ID 787939, (2011), 7 pages.

[16] S. D. Purohit and R. K. Raina, "Chebyshev type inequalities for the saigo fractional integrals and their q-analogues," Journal of Mathematical Inequalities, vol. 7, no. 2, (2013), pp. 239-249.

[17] J. D. Ramīrez and A. S. Vatsala, "Monotonic iterative technique for fractional differential equations with periodic boundary conditions," Opuscula Mathematica, vol. 29, no.3,(2009), pp. 289-304.

[18] W. T. Sulaiman, "Some new fractional integral inequalities," Journal Mathematical Analysis, vol. 2, no. 2, 2011, pp. 23-28.

[19] M. Saigo, "A remark on integral operators involving the Gauss hypergeometric functions," Mathematical Reports, Kyushu University, vol. 11, (1978), pp. 135-143.

[20] M.-Z. Sarıkaya, and H. Ogunmez, On new inequalities via RiemannLiouville Fractional Integration, Abstract and Applied Analysis, vol., Article ID 428983, (2012), 10 pages.

[21] S.-G.Samko, A.-A. Kilbas, and O.-I. Marichev, Fractional Integral and Derivatives, Theory and Applications, Gordon and Breach, Yverdon et alibi. (1993). 
[22] H. Yıldırım, Z. Kırtay, "Ostrowski Inequality for Generalized Fractional Integral and Related Inequalities" ,Malaya J. Mat. 2(3) (2014), 322-329. 